\title{
The nearest symmetric fuzzy solution for a symmetric fuzzy linear system
}

\author{
T. Allahviranloo, E. Haghi, M. Ghanbari
}

\begin{abstract}
In this paper, the nearest symmetric fuzzy solution for a symmetric $L$ - $L$ fuzzy linear system ( $S$ - $L$-FLS) is obtained by a new metric. To this end, the $S$ - $L$-FLS is transformed to the non-linear programming problem (NLP). The solution of the obtained NLP is our favorite fuzzy number vector solution. Also, it is shown that if an $S$ - $L$-FLS has unique fuzzy solution, then its solution is symmetric. Two constructive algorithms are presented in details and the method is illustrated by solving several numerical examples.
\end{abstract}

\section{Introduction}

One field of applied mathematics that play a major role in various areas of sciences is solving systems of linear equations where some of the system's parameters are proposed as fuzzy numbers. Therefore, it is natural that we want to develop numerical procedures to solve such systems.

A general model for solving an $n \times n$ fuzzy linear system (FLS) which coefficient matrix is crisp and the right hand side column is arbitrary fuzzy number vector were proposed by Friedman et al. [16]. Based on Friedman et al.'s method, Allahviranloo $[2,3,5,8]$ used the various numerical methods to solve fuzzy linear systems. Recently, he and Salahshour [10] have proposed a simple and practical method to obtain symmetric fuzzy solutions of fuzzy linear

Key Words: Symmetric $L-L$ fuzzy number; Symmetric $L-L$ fuzzy linear system; Nonlinear programming problem; Modified Euclidean distance.

2010 Mathematics Subject Classification: 65F99, 65K99, 93C42, 03E72, 28E10

Received: February, 2011.

Revised: June, 2011.

Accepted: February, 2012 
systems. Also, Ghanbari and his colleague [17] have proposed an approach for computing the general compromised solution of an $L$ - $R$ fuzzy linear system by use of a ranking function when the coefficient matrix is a crisp $m \times n$ matrix. In continuation to these works, Dehghan et al. $[12,13]$ have discussed the case in which all parameters in a fuzzy linear system are fuzzy numbers, which they called it a fully fuzzy linear system, and extend some iterative methods on the this system. Recently, Allahviranloo and Salahshour [11] have proposed a new method to obtain symmetric solution of fully fuzzy linear system based on 1-cut expansion. For more research papers see $[1,6,7,15]$.

In this paper, we extend Diamond's idea [14] to define a new metric distance for $L-R$ fuzzy numbers with the fixed shape functions $L(\cdot)$ and $R(\cdot)$. Then, we use the proposed metric distance to introduce a method for obtaining the nearest symmetric fuzzy solution for symmetric $L-L$ fuzzy linear system $(S$ $L$-FLS), where the coefficient matrix is a crisp $n \times n$ matrix and the right hand side column is a symmetric $L-L$ fuzzy number vector with the fixed $L(\cdot)$ and $R(\cdot)$. Also, it is shown that if an $S$ - $L$-FLS has unique fuzzy solution, then its solution is symmetric. According to our method, for solving $S$ - $L$ FLS, we construct a minimization problem based on the proposed metric and then solve it. If the optimal value is zero, then we obtain a symmetric exact solution. Otherwise, $S$ - $L$-FLS has not any exact solution and thus we compute a symmetric approximate solution for it.

The structure of this paper is organized as follows. In Section 2 we present some basic definitions and results on $L-R$ fuzzy numbers. In Section 3 we introduce a metric distance and verify some its properties. In Section 4 we define an $S$ - $L$-FLS and present two algorithms for solving it. In Section 5 we give some numerical examples. Conclusion is drawn in Section 6.

\section{Preliminaries}

The basic definition of fuzzy numbers is given in [18].

Definition 2.1. A fuzzy subset $\widetilde{A}$ of the real line $\mathbb{R}$ with membership function $\mu_{\widetilde{A}}: \mathbb{R} \rightarrow[0,1]$ is called a fuzzy number if

(1) $\mu_{\widetilde{A}}$ is upper semi-continuous,

(2) $\mu_{\widetilde{A}}(x)=0$ outside some interval $[c, d]$,

(3) There are real numbers $a$ and $b$ such that $c \leqslant a \leqslant b \leqslant d$ and

i. $\mu_{\widetilde{A}}(x)$ is monotonic increasing on $[c, a]$,

ii. $\mu_{\widetilde{A}}(x)$ is monotonic decreasing on $[b, d]$,

iii. $\mu_{\widetilde{A}}(x)=1, a \leqslant x \leqslant b$.

The support and core of $\widetilde{A}$ are defined by the sets $S(\widetilde{A})=\overline{\left\{x \in \mathbb{R}: \mu_{\widetilde{A}}(x)>0\right\}}$ and $C(\widetilde{A})=\left\{x \in \mathbb{R}: \mu_{\widetilde{A}}(x)=1\right\}$, respectively. Then, based on the Definition 
2.1 , we have

$$
S(\widetilde{A})=[c, d], \quad C(\widetilde{A})=[a, b] .
$$

Definition 2.2. An $L-R$ fuzzy number $\widetilde{A}=\left(a_{1}, a_{2} ; \alpha, \beta\right)_{L R}, a_{1} \leqslant a_{2}$, $\alpha, \beta>0$, is defined as follows:

$$
\mu_{\widetilde{A}}(x)= \begin{cases}L\left(\frac{a_{1}-x}{\alpha}\right), & x \leqslant a_{1}, \\ 1, & a_{1} \leqslant x \leqslant a_{2}, \\ R\left(\frac{x-a_{2}}{\beta}\right), & a_{2} \leqslant x,\end{cases}
$$

where $a_{1}$ and $a_{2}$ are the left and right points of core, respectively; $\alpha$ and $\beta$ are the left and right spreads, respectively; and the functions $L(\cdot)$ and $R(\cdot)$, which are called left and right shape functions, satisfying:

(1) $L(\cdot)$ and $R(\cdot)$ are non-increasing from $\mathbb{R}^{+}$to $[0,1]$,

(2) $L(0)=R(0)=1, \quad L(1)=R(1)=0$.

Remark 2.3. If for fuzzy number $\widetilde{A}$, at least one of left or right spreads be zero, then we extend the Definition 2.2 as follows:

$$
\begin{aligned}
& \text { if } \widetilde{A}=\left(a_{1}, a_{2} ; 0, \beta\right)_{L R} \quad \text { then } \mu_{\widetilde{A}}(x)= \begin{cases}0, & x<a_{1}, \\
1, & a_{1} \leqslant x \leqslant a_{2}, \\
R\left(\frac{x-a_{2}}{\beta}\right), & a_{2} \leqslant x,\end{cases} \\
& \text { if } \widetilde{A}=\left(a_{1}, a_{2} ; \alpha, 0\right)_{L R} \text { then } \mu_{\widetilde{A}}(x)= \begin{cases}L\left(\frac{a_{1}-x}{\alpha}\right), & x \leqslant a_{1}, \\
1, & a_{1} \leqslant x \leqslant a_{2}, \\
0, & a_{2}<x,\end{cases} \\
& \text { if } \widetilde{A}=\left(a_{1}, a_{2} ; 0,0\right)_{L R} \text { then } \mu_{\widetilde{A}}(x)= \begin{cases}0, & x<a_{1}, \\
1, & a_{1} \leqslant x \leqslant a_{2}, \\
0, & a_{2}<x .\end{cases}
\end{aligned}
$$

Thus, by Remark 2.3, the crisp numbers and the crisp intervals can be considered as $L-R$ fuzzy numbers. In particular, when $L(\cdot)$ and $R(\cdot)$ are linear functions $(L(x)=R(x)=\max \{0,1-x\})$ and $a_{1}<a_{2}$, fuzzy number $\widetilde{A}$ denotes trapezoidal fuzzy number. Also, when $L(\cdot)$ and $R(\cdot)$ are linear functions $(L(x)=R(x)=\max \{0,1-x\})$ and $a_{1}=a_{2}$, fuzzy number $\widetilde{A}$ denotes triangular fuzzy number. Clearly, for $\widetilde{A}=\left(a_{1}, a_{2} ; \alpha, \beta\right)_{L R}$, we have

$$
S(\widetilde{A})=\left[a_{1}-\alpha, a_{2}+\beta\right], \quad C(\widetilde{A})=\left[a_{1}, a_{2}\right] .
$$

Definition 2.4. An $L-R$ fuzzy number $\widetilde{A}=\left(a_{1}, a_{2} ; \alpha, \beta\right)_{L R}$ is called a symmetric fuzzy number if $\alpha=\beta$ and $L(x)=R(x)$ for all $x \in \mathbb{R}^{+}$. In this case, we say $\widetilde{A}$ is a symmetric $L-L$ fuzzy number. 
Definition 2.5. Two L-R fuzzy numbers

$$
\widetilde{A}=\left(a_{1}, a_{2} ; \alpha, \beta\right)_{L R} \quad \text { and } \quad \widetilde{B}=\left(b_{1}, b_{2} ; \gamma, \eta\right)_{L R}
$$

are said to be equal, if and only if $a_{1}=b_{1}, a_{2}=b_{2}, \alpha=\gamma$ and $\beta=\eta$.

Definition 2.6. For L-R fuzzy numbers, addition and scalar multiplication are defined as follows:

$$
\begin{gathered}
\widetilde{A}+\widetilde{B}=\left(a_{1}, a_{2} ; \alpha, \beta\right)_{L R}+\left(b_{1}, b_{2} ; \gamma, \eta\right)_{L R}=\left(a_{1}+b_{1}, a_{2}+b_{2} ; \alpha+\gamma, \beta+\eta\right)_{L R}, \\
\lambda \cdot \widetilde{A}=\lambda \cdot\left(a_{1}, a_{2} ; \alpha, \beta\right)_{L R}= \begin{cases}\left(\lambda a_{1}, \lambda a_{2} ; \lambda \alpha, \lambda \beta\right)_{L R}, & \lambda \geqslant 0, \\
\left(\lambda a_{2}, \lambda a_{1} ;-\lambda \beta,-\lambda \alpha\right)_{R L}, & \lambda<0 .\end{cases}
\end{gathered}
$$

Note that we use a fixed function $L(\cdot)$ and a fixed function $R(\cdot)$ for all fuzzy numbers in each problem. Then, through the end of this paper, we eliminate the subscript " $L R$ " in representation of $L-R$ fuzzy numbers.

\section{$3 \quad$ A metric distance for $L-R$ fuzzy numbers}

Now, we are going to define a distance between two $L-R$ fuzzy numbers by modification of the Euclidean distance.

Definition 3.1. For two interval numbers $A=\left[a_{1}, a_{2}\right]$ and $B=\left[b_{1}, b_{2}\right]$ we define the modified Euclidean distance between them as

$$
d_{M E}(A, B)=\sqrt{\frac{\left(a_{1}-b_{1}\right)^{2}+\left(a_{2}-b_{2}\right)^{2}}{2}},
$$

or

$$
d_{M E}^{2}(A, B)=\frac{\left(a_{1}-b_{1}\right)^{2}+\left(a_{2}-b_{2}\right)^{2}}{2} .
$$

Theorem 3.2. The function $d_{M E}$ is a metric for the family of all interval numbers.

Proof. Since $d_{M E}(A, B)=\frac{\sqrt{2}}{2} d_{E}(A, B)$, where

$$
d_{E}(A, B)=\sqrt{\left(a_{1}-b_{1}\right)^{2}+\left(a_{2}-b_{2}\right)^{2}},
$$

is Euclidean metric for interval numbers, it is clear that $d_{M E}$ is a metric too.

Obviously, when $a_{1}=a_{2}=a$ and $b_{1}=b_{2}=b$, we conclude

$$
d_{M E}(A, B)=|a-b| \text {, }
$$

but,

$$
d_{E}(A, B) \neq|a-b|
$$


Therefore, the function $d_{M E}$ preserve the traditional distance in real space, while the function $d_{E}$ has not such property, that is why we call it "modified Euclidean distance". We now use $d_{M E}$ to introduce a metric distance for the set of $L-R$ fuzzy numbers.

Definition 3.3. Let the shape functions $L(\cdot)$ and $R(\cdot)$ are fixed. Consider two $L-R$ fuzzy numbers as $\widetilde{A}=\left(a_{1}, a_{2} ; \alpha, \beta\right)$ and $\widetilde{B}=\left(b_{1}, b_{2} ; \gamma, \eta\right)$. We define the distance between $\widetilde{A}$ and $\widetilde{B}$ as follows:

$$
d(\widetilde{A}, \widetilde{B})=\sqrt{\frac{d_{M E}^{2}(S(\widetilde{A}), S(\widetilde{B}))+d_{M E}^{2}(C(\widetilde{A}), C(\widetilde{B}))}{2}},
$$

or

$$
d^{2}(\widetilde{A}, \widetilde{B})=\frac{d_{M E}^{2}(S(\widetilde{A}), S(\widetilde{B}))+d_{M E}^{2}(C(\widetilde{A}), C(\widetilde{B}))}{2} .
$$

Then, by Eqs. (2) and (4) we have

$d^{2}(\widetilde{A}, \widetilde{B})=\frac{\left[\left(a_{1}-b_{1}\right)-(\alpha-\gamma)\right]^{2}+\left[\left(a_{2}-b_{2}\right)+(\beta-\eta)\right]^{2}+\left(a_{1}-b_{1}\right)^{2}+\left(a_{2}-b_{2}\right)^{2}}{4}$.

Remark 3.4. If $\widetilde{A}$ and $\widetilde{B}$ are two crisp numbers, i.e., $\widetilde{A}=(a, a ; 0,0)$ and $\widetilde{B}=(b, b ; 0,0)$, then the our metric is equivalent to the traditional metric in real space, namely

$$
d(\widetilde{A}, \widetilde{B})=|a-b| .
$$

Remark 3.5. If $\widetilde{A}$ and $\widetilde{B}$ are two crisp interval numbers, i.e., $\widetilde{A}=\left(a_{1}, a_{2} ; 0,0\right)$ and $\widetilde{B}=\left(b_{1}, b_{2} ; 0,0\right)$, then the proposed metric is equivalent to the modified Euclidean metric, namely

$$
d(\widetilde{A}, \widetilde{B})=d_{M E}\left(\left[a_{1}, a_{2}\right],\left[b_{1}, b_{2}\right]\right) .
$$

We will show that the function $d$ defined in Eq. (3) is a metric for the set of $L-R$ fuzzy numbers with the fixed $L(\cdot)$ and $R(\cdot)$. To this end, we have

Lemma 3.6. If $a, b, c, d \in \mathbb{R}$, then $(a b+c d) \leqslant \sqrt{a^{2}+c^{2}} \cdot \sqrt{b^{2}+d^{2}}$.

Proof. Since

$$
\left(a^{2}+c^{2}\right) \cdot\left(b^{2}+d^{2}\right)-(a b+c d)^{2}=a^{2} d^{2}+c^{2} b^{2}-2 a d c b=(a d-c b)^{2} \geqslant 0,
$$

the proof is completed.

Lemma 3.7. If $a, b, c, d \in \mathbb{R}$, then $(a+b)^{2}+(c+d)^{2} \leqslant\left(\sqrt{a^{2}+c^{2}}+\sqrt{b^{2}+d^{2}}\right)^{2}$. 
Proof. Since

$\left(\sqrt{a^{2}+c^{2}}+\sqrt{b^{2}+d^{2}}\right)^{2}-(a+b)^{2}-(c+d)^{2}=2\left(\sqrt{a^{2}+c^{2}} \cdot \sqrt{b^{2}+d^{2}}-(a b+c d)\right)$, by Lemma 3.6, the proof is completed.

Theorem 3.8. The function d defined in Eq. (3) is a metric for the family of all $L-R$ fuzzy numbers with fixed $L(\cdot)$ and $R(\cdot)$.

Proof. According to Theorem 3.2, since the function $d_{M E}$ is a metric for the family of all interval numbers, then it is easy to verify that the function $d$ satisfies the following properties:

1) $d(\widetilde{A}, \widetilde{B}) \geqslant 0$,

2) $d(\widetilde{A}, \widetilde{B})=0 \Leftrightarrow \widetilde{A}=\widetilde{B}$,

3) $d(\widetilde{A}, \widetilde{B})=d(\widetilde{B}, \widetilde{A})$.

Note that since the functions $L(\cdot)$ and $R(\cdot)$ are fixed, property 2 is holds. It is only remained to show that the triangle inequality property is holds. By virtue of Theorem 3.2 and Lemma 3.7, for three $L$ - $R$ fuzzy numbers $\widetilde{A}, \widetilde{B}$ and $\widetilde{C}$ we have

$$
\begin{aligned}
d^{2}(\widetilde{A}, \widetilde{C}) & =\frac{d_{M E}^{2}(S(\widetilde{A}), S(\widetilde{C}))}{2}+\frac{d_{M E}^{2}(C(\widetilde{A}), C(\widetilde{C}))}{2} \\
& \leqslant \frac{\left(d_{M E}(S(\widetilde{A}), S(\widetilde{B}))+d_{M E}(S(\widetilde{B}), S(\widetilde{C}))\right)^{2}}{2} \\
& +\frac{\left(d_{M E}(C(\widetilde{A}), C(\widetilde{B}))+d_{M E}(C(\widetilde{B}), C(\widetilde{C}))\right)^{2}}{2} \\
& \leqslant(d(\widetilde{A}, \widetilde{B})+d(\widetilde{B}, \widetilde{C}))^{2} .
\end{aligned}
$$

Remark 3.9. It is clear that there exist distinct fuzzy numbers $\widetilde{A}$ and $\widetilde{B}$ such that $d(\widetilde{A}, \widetilde{B})=0$, and so the function d defined in Eq. (3) is not a metric for the family of all fuzzy numbers. For example, consider the fuzzy numbers $\widetilde{A}$ and $\widetilde{B}$ indicated in Fig. 1. Since $L(\cdot)$ and $R(\cdot)$ are not fixed, then $\widetilde{A} \neq \widetilde{B}$ while $d(\widetilde{A}, \widetilde{B})=0$. In fact, the function $d$ is a pseudo-metric for family of all fuzzy numbers. Therefore, in Theorem 3.8, it is necessary that two shape functions $L(\cdot)$ and $R(\cdot)$ are fixed.

Theorem 3.10. For the fixed shape functions $L(\cdot)$ and $R(\cdot)$, the metric $d$ satisfies the following properties:

1) if $L(x)=R(x), \forall x \in \mathbb{R}^{+}$, then $d(\widetilde{A}-\widetilde{C}, \widetilde{B}-\widetilde{C})=d(\widetilde{A}, \widetilde{B})$,

2) $d(\widetilde{A}+\widetilde{C}, \widetilde{B}+\widetilde{C})=d(\widetilde{A}, \widetilde{B})$,

3) $\quad d(\lambda \widetilde{A}, \lambda \widetilde{B})=|\lambda| d(\widetilde{A}, \widetilde{B}), \quad \forall \lambda \in \mathbb{R}$, 


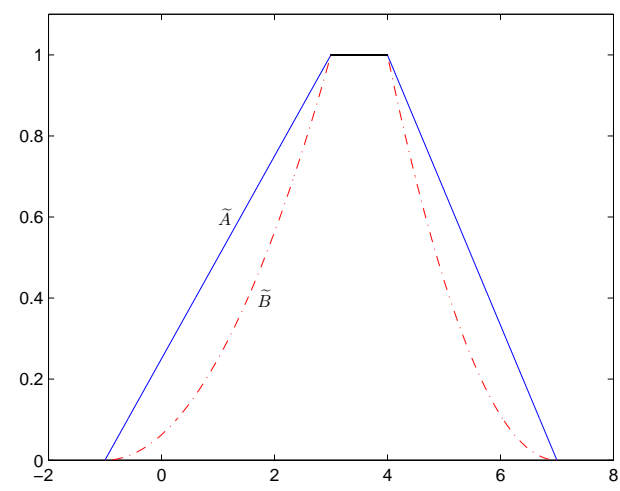

Fig. 1. The fuzzy numbers $\widetilde{A}$ and $\widetilde{B}$.

where $\widetilde{A}, \widetilde{B}$ and $\widetilde{C}$ are $L-R$ fuzzy numbers and $\widetilde{A}-\widetilde{C}=\widetilde{A}+(-1) \widetilde{C}$.

Proof. 1) Let $\widetilde{A}=\left(a_{1}, a_{2} ; \alpha, \beta\right), \widetilde{B}=\left(b_{1}, b_{2} ; \gamma, \eta\right)$ and $\widetilde{C}=\left(c_{1}, c_{2} ; \delta, \theta\right)$. Then we have

$\begin{array}{ll}S(\widetilde{A}-\widetilde{C})=\left[a_{1}-c_{2}-\alpha-\theta, a_{2}-c_{1}+\beta+\delta\right], & C(\widetilde{A}-\widetilde{C})=\left[a_{1}-c_{2}, a_{2}-c_{1}\right], \\ S(\widetilde{B}-\widetilde{C})=\left[b_{1}-c_{2}-\gamma-\theta, b_{2}-c_{1}+\eta+\delta\right], & C(\widetilde{B}-\widetilde{C})=\left[b_{1}-c_{2}, b_{2}-c_{1}\right] .\end{array}$

It can be easily verified that

$$
d_{M E}(S(\widetilde{A}-\widetilde{C}), S(\widetilde{B}-\widetilde{C}))=d_{M E}(S(\widetilde{A}), S(\widetilde{B})),
$$

and

$$
d_{M E}(C(\widetilde{A}-\widetilde{C}), C(\widetilde{B}-\widetilde{C}))=d_{M E}(C(\widetilde{A}), C(\widetilde{B})) .
$$

Thus

$$
d(\widetilde{A}-\widetilde{C}, \widetilde{B}-\widetilde{C})=d(\widetilde{A}, \widetilde{B}) .
$$

Note that if $L(x) \neq R(x)$, then by Definition $2.5,(-1) \widetilde{C}$ is a $R-L$ fuzzy number and thus we can not use the addition formula presented in Definition 2.5.

2) The proof is similar to the first part.

3) At first, let $\lambda<0$. Therefore, we will have

$$
S(\lambda \widetilde{A})=\left[\lambda a_{2}+\lambda \beta, \lambda a_{1}-\lambda \alpha\right], \quad C(\lambda \widetilde{A})=\left[\lambda a_{2}, \lambda a_{1}\right],
$$




$$
S(\lambda \widetilde{B})=\left[\lambda b_{2}+\lambda \eta, \lambda b_{1}-\lambda \gamma\right], \quad C(\lambda \widetilde{B})=\left[\lambda b_{2}, \lambda b_{1}\right] .
$$

Hence

$$
\begin{aligned}
d_{M E}^{2}(S(\lambda \widetilde{A}), S(\lambda \widetilde{B})) & =\lambda^{2} \frac{\left[\left(a_{2}-b_{2}\right)+(\beta-\eta)\right]^{2}+\left[\left(a_{1}-b_{1}\right)-(\alpha-\gamma)\right]^{2}}{2} \\
& =\lambda^{2} d_{M E}^{2}(S(\widetilde{A}), S(\widetilde{B})) .
\end{aligned}
$$

Thus

$$
d_{M E}(S(\lambda \widetilde{A}), S(\lambda \widetilde{B}))=|\lambda| d_{M E}(S(\widetilde{A}), S(\widetilde{B})),
$$

and similarly

$$
d_{M E}(C(\lambda \widetilde{A}), C(\lambda \widetilde{B}))=|\lambda| d_{M E}(C(\widetilde{A}), C(\widetilde{B})) .
$$

Therefore

$$
d_{M E}(\lambda \widetilde{A}, \lambda \widetilde{B})=|\lambda| d_{M E}(\widetilde{A}, \widetilde{B}) .
$$

The proof for $\lambda \geqslant 0$ is easy and omitted.

Definition 3.11. A vector $\widetilde{X}=\left(\widetilde{x_{1}}, \widetilde{x_{1}}, \ldots, \widetilde{x_{n}}\right)^{T}$, where $\widetilde{x_{i}}, 1 \leqslant i \leqslant n$ are $L-R$ fuzzy numbers, is called an $L-R$ fuzzy vector.

Definition 3.12. For two $L-R$ fuzzy vectors $\widetilde{X}=\left(\widetilde{x_{1}}, \widetilde{x_{2}}, \ldots, \widetilde{x_{n}}\right)^{T}$ and $\widetilde{Y}=\left(\widetilde{y_{1}}, \widetilde{y_{2}}, \ldots, \widetilde{y_{n}}\right)^{T}$, we define

$$
D_{p}(\widetilde{X}, \widetilde{Y})=\left(\sum_{i=1}^{n} d^{p}\left(\widetilde{x_{i}}, \widetilde{y}_{i}\right)\right)^{\frac{1}{p}}
$$

as distance between them, where $p \geqslant 1$.

In this paper, for obtaining the distance between two $L-R$ fuzzy vectors we consider $p=2$ in Definition 3.12. In the next section, we define a symmetric $L-L$ FLS and use the our metric to find either the symmetric exact solution or the symmetric approximate solution for the such system.

\section{Solving symmetric $L-L$ fuzzy linear system}

Definition 4.1. Let the shape function $L(\cdot)$ is fixed. The $n \times n$ linear system

$$
\left\{\begin{array}{l}
a_{11} \widetilde{x_{1}}+a_{12} \widetilde{x_{2}}+\cdots+a_{1 n} \widetilde{x_{n}}=\widetilde{b_{1}} \\
a_{21} \widetilde{x_{1}}+a_{22} \widetilde{x_{2}}+\cdots+a_{2 n} \widetilde{x_{n}}=\widetilde{b_{2}} \\
\vdots \\
a_{n 1} \widetilde{x_{1}}+a_{n 2} \widetilde{x_{2}}+\cdots+a_{n n} \widetilde{x_{n}}=\widetilde{b_{n}}
\end{array}\right.
$$


where the coefficient matrix $A=\left(a_{i j}\right), 1 \leqslant i, j \leqslant n$ is a crisp $n \times n$ matrix and $\widetilde{b_{i}}, 1 \leqslant i \leqslant n$ are symmetric $L-L$ fuzzy numbers, is called a symmetric $L-L$ fuzzy linear system $(S-L-F L S)$.

The matrix form of the above equations is

$$
A \widetilde{X}=\widetilde{B}
$$

where $\widetilde{X}=\left(\widetilde{x_{1}}, \widetilde{x_{2}}, \ldots, \widetilde{x_{n}}\right)^{T}, \widetilde{B}=\left(\widetilde{b_{1}}, \widetilde{b_{2}}, \ldots, \widetilde{b_{n}}\right)^{T}$ and $\widetilde{x_{i}}$ and $\widetilde{b_{i}}$ are $L-L$ fuzzy numbers as follows:

$$
\widetilde{x_{i}}=\left(x_{1}^{i}, x_{2}^{i} ; \alpha_{x}^{i}, \beta_{x}^{i}\right), \quad \widetilde{b_{i}}=\left(b_{1}^{i}, b_{2}^{i} ; \alpha_{b}^{i}, \alpha_{b}^{i}\right), \quad i=1,2, \ldots, n .
$$

Definition 4.2. We say $\widetilde{X}=\left(\widetilde{x_{1}}, \widetilde{x_{2}}, \ldots, \widetilde{x_{n}}\right)^{T}$ is a solution for $S-L-F L S$ (7) if and only if

$$
\sum_{j=1}^{n} a_{i j} \widetilde{x_{j}}=\widetilde{b_{i}}, \quad i=1,2, \ldots, n,
$$

where $\widetilde{x_{j}}, 1 \leqslant j \leqslant n$ are fuzzy numbers.

Theorem 4.3. If $\widetilde{X}=\left(\widetilde{x_{1}}, \widetilde{x_{2}}, \ldots, \widetilde{x_{n}}\right)^{T}$ be unique solution of $S-L-F L S$ (7), then $\widetilde{X}$ is a symmetric $L-L$ fuzzy number vector, i.e., $\widetilde{x_{j}}, 1 \leqslant j \leqslant n$ are symmetric $L-L$ fuzzy numbers.

Proof. It is sufficient to show that $\alpha_{x}^{i}=\beta_{x}^{i}$, for all $i=1,2, \ldots, n$. Since $\widetilde{X}$ is a solution for S- $L$-FLS $(7)$, then

$$
\sum_{j=1}^{n} a_{i j} \widetilde{x_{j}}=\widetilde{b_{i}}, \quad i=1,2, \ldots, n .
$$

Consequently

$$
\begin{aligned}
& \sum_{j \in \Gamma_{i}^{+}} a_{i j} \alpha_{x}^{j}-\sum_{j \in \Gamma_{i}^{-}} a_{i j} \beta_{x}^{j}=\alpha_{b}^{i}, \\
& \sum_{j \in \Gamma_{i}^{+}} a_{i j} \beta_{x}^{j}+\sum_{j \in \Gamma_{i}^{-}} a_{i j} \alpha_{x}^{j}=\alpha_{b}^{i},
\end{aligned}
$$

for all $i=1,2, \ldots, n$, where

$$
\begin{aligned}
& \Gamma_{i}^{+}=\left\{j: 1 \leqslant j \leqslant n, a_{i j} \geqslant 0\right\}, \\
& \Gamma_{i}^{-}=\left\{j: 1 \leqslant j \leqslant n, a_{i j}<0\right\} .
\end{aligned}
$$


From Eqs. (11) and (12) we have

$$
\sum_{j \in \Gamma_{i}^{+}} a_{i j}\left(\alpha_{x}^{j}-\beta_{x}^{j}\right)+\sum_{j \in \Gamma_{i}^{-}} a_{i j}\left(\alpha_{x}^{j}-\beta_{x}^{j}\right)=0, \quad i=1,2, \ldots, n .
$$

Setting

$$
V=\left(v_{1}, v_{2}, \ldots, v_{n}\right)^{T}, \quad v_{j}=\alpha_{x}^{j}-\beta_{x}^{j}, \quad j=1,2, \ldots, n,
$$

we have

$$
A V=0 .
$$

Since system (7) has unique solution, then the matrix $A$ is nonsingular [16] and therefore $V=0$, i.e., $v_{j}=0$, for all $j=1,2, \ldots, n$.

Thus, the proof is completed.

Now, suppose that the $\widetilde{X}=\left(\widetilde{x_{1}}, \widetilde{x_{2}}, \ldots, \widetilde{x_{n}}\right)^{T}$ is unique solution of S- $L$-FLS (7), then we have

$$
\begin{aligned}
& \sum_{j \in \Gamma_{i}^{+}} a_{i j} x_{1}^{j}+\sum_{j \in \Gamma_{i}^{-}} a_{i j} x_{2}^{j}=b_{1}^{i}, \\
& \sum_{j \in \Gamma_{i}^{+}} a_{i j} x_{2}^{j}+\sum_{j \in \Gamma_{i}^{-}} a_{i j} x_{1}^{j}=b_{2}^{i}, \\
& \sum_{j \in \Gamma_{i}^{+}} a_{i j} \alpha_{x}^{j}-\sum_{j \in \Gamma_{i}^{-}} a_{i j} \alpha_{x}^{j}=\alpha_{b}^{i},
\end{aligned}
$$

where the sets $\Gamma_{i}^{+}$and $\Gamma_{i}^{-}$are defined in Eqs. (13) and (14), respectively. Also, note that according to Theorem $4.3, \widetilde{X}$ is a symmetric $L$ - $L$ fuzzy number vector. We set

$$
\Lambda_{x}=\left(\alpha_{x}^{1}, \alpha_{x}^{2}, \ldots, \alpha_{x}^{n}\right)^{T}, \quad \Lambda_{b}=\left(\alpha_{b}^{1}, \alpha_{b}^{2}, \ldots, \alpha_{b}^{n}\right)^{T},
$$

and

$$
W=\left(w_{1}, w_{2}, \ldots, w_{n}\right)^{T}, \quad C=\left(c_{1}, c_{2}, \ldots, c_{n}\right)^{T},
$$

where

$$
w_{i}=x_{1}^{i}+x_{2}^{i}, \quad c_{i}=b_{1}^{i}+b_{2}^{i}, \quad i=1,2, \ldots, n .
$$

Therefore, from Eqs. (16)-(18) we have

$$
A W=C, \quad|A| \Lambda_{x}=\Lambda_{b} .
$$


Since S- $L$-FLS (7) has unique solution, then the matrices $A$ and $|A|$ are nonsingular [16]. Therefore, by solving the systems (19) we obtain the vectors $W$ and $\Lambda_{x}$ and consequently we will have

$$
x_{2}^{i}=w_{i}-x_{1}^{i}, \quad i=1,2, \ldots, n .
$$

On the other hand, It is clear that if $\widetilde{X}$ be an exact solution of S- $L$-FLS (7), then

$$
d\left((A \widetilde{X})_{i}, \widetilde{b_{i}}\right)=0, \quad i=1,2, \ldots, n .
$$

Therefore, a logical way to find a symmetric $L-L$ fuzzy vector solution is to minimization distance between the vectors $A \widetilde{X}$ and $\widetilde{B}$. To this end, we set

$$
(A \widetilde{X})_{i}=\widetilde{y}_{i}=\left(y_{1}^{i}, y_{2}^{i} ; \alpha_{y}^{i}, \alpha_{y}^{i}\right)
$$

Hence

$$
\begin{gathered}
y_{1}^{i}=\sum_{j \in \Gamma_{i}^{+}} a_{i j} x_{1}^{j}+\sum_{j \in \Gamma_{i}^{-}} a_{i j} x_{2}^{j}, \quad y_{2}^{i}=\sum_{j \in \Gamma_{i}^{+}} a_{i j} x_{2}^{j}+\sum_{j \in \Gamma_{i}^{-}} a_{i j} x_{1}^{j}, \\
\alpha_{y}^{i}=\sum_{j \in \Gamma_{i}^{+}} a_{i j} \alpha_{x}^{j}-\sum_{j \in \Gamma_{i}^{-}} a_{i j} \alpha_{x}^{j} .
\end{gathered}
$$

By Definition 3.12 and using the above notations we define the following minimization problem to find a symmetric $L-L$ fuzzy vector solution,

$$
\begin{cases}\min & Z=\sum_{i=1}^{n} d^{2}\left(\widetilde{y}_{i}, \widetilde{b}_{i}\right) \\ \text { s.t. } & \\ & x_{1}^{i} \leqslant x_{2}^{i}, \\ & \alpha_{x}^{i} \geqslant \max \left\{0, \alpha_{x}^{* i}\right\}, \\ & x_{1}^{i}, x_{2}^{i}: \text { free }, \quad i=1,2, \ldots, n\end{cases}
$$

where

$$
\begin{aligned}
d^{2}\left(\widetilde{y}_{i}, \widetilde{b}_{i}\right) & =\frac{\left[\left(y_{1}^{i}-b_{1}^{i}\right)-\left(\alpha_{y}^{i}-\alpha_{b}^{i}\right)\right]^{2}+\left[\left(y_{2}^{i}-b_{2}^{i}\right)+\left(\alpha_{y}^{i}-\alpha_{b}^{i}\right)\right]^{2}}{4} \\
& +\frac{\left(y_{1}^{i}-b_{1}^{i}\right)^{2}+\left(y_{2}^{i}-b_{2}^{i}\right)^{2}}{4}
\end{aligned}
$$

and $y_{1}^{i}, y_{2}^{i}$ and $\alpha_{y}^{i}$ are defined in Eqs. (23) and (24) and also

$$
\Lambda_{x}^{*}=\left(\alpha_{x}^{* 1}, \alpha_{x}^{* 2}, \ldots, \alpha_{x}^{* n}\right)^{T},
$$

is unique solution of system $A \Lambda_{x}=\Lambda_{b}$. 
On the other hand, from Eqs. (20), (23) and (24), we conclude

$$
\begin{gathered}
y_{1}^{i}=\sum_{j \in \Gamma_{i}^{+}} a_{i j} x_{1}^{j}-\sum_{j \in \Gamma_{i}^{-}} a_{i j} x_{1}^{j}+\sum_{j \in \Gamma_{i}^{-}} a_{i j} w_{j}, \\
y_{2}^{i}=\sum_{j \in \Gamma_{i}^{+}} a_{i j} w_{j}+\sum_{j \in \Gamma_{i}^{-}} a_{i j} x_{1}^{j}-\sum_{j \in \Gamma_{i}^{+}} a_{i j} x_{1}^{j}, \\
\alpha_{y}^{i}=\sum_{j \in \Gamma_{i}^{+}} a_{i j} \alpha_{x}^{j}-\sum_{j \in \Gamma_{i}^{-}} a_{i j} \alpha_{x}^{j},
\end{gathered}
$$

Note that in the above equations (26)-(28) we omitted the parameter $x_{2}^{i}$. Now, in the minimization problem $(2.24)$, we let

$$
\begin{array}{ll}
\Omega_{1}^{i}:=\left(y_{1}^{i}-b_{1}^{i}\right)-\left(\alpha_{y}^{i}-\alpha_{b}^{i}\right), & \Omega_{2}^{i}:=\left(y_{2}^{i}-b_{2}^{i}\right)+\left(\alpha_{y}^{i}-\alpha_{b}^{i}\right), \\
\Omega_{3}^{i}:=\left(y_{1}^{i}-b_{1}^{i}\right), & \Omega_{4}^{i}:=\left(y_{2}^{i}-b_{2}^{i}\right) .
\end{array}
$$

From Eqs. (2.26)-(2.28) we obtain

$$
\begin{aligned}
\Omega_{1}^{i} & =\sum_{j \in \Gamma_{i}^{+}} a_{i}\left(x_{1}^{j}-\alpha_{x}^{j}\right)-\sum_{j \in \Gamma_{i}^{-}} a_{i j}\left(x_{1}^{j}-\alpha_{x}^{j}\right)+\sum_{j \in \Gamma_{i}^{-}} a_{i j} w_{j}-\left(b_{1}^{i}-\alpha_{b}^{i}\right), \\
\Omega_{2}^{i} & =\sum_{j \in \Gamma_{i}^{-}} a_{i j}\left(x_{1}^{j}-\alpha_{x}^{j}\right)-\sum_{j \in \Gamma_{i}^{+}} a_{i j}\left(x_{1}^{j}-\alpha_{x}^{j}\right)+\sum_{j \in \Gamma_{i}^{+}} a_{i j} w_{j}-\left(b_{2}^{i}+\alpha_{b}^{i}\right), \\
\Omega_{3}^{i} & =\sum_{j \in \Gamma_{i}^{+}} a_{i j} x_{1}^{j}-\sum_{j \in \Gamma_{i}^{-}} a_{i j} x_{1}^{j}+\sum_{j \in \Gamma_{i}^{-}} a_{i j} w_{j}-b_{1}^{i}, \\
\Omega_{4}^{i} & =\sum_{j \in \Gamma_{i}^{-}} a_{i j} x_{1}^{j}-\sum_{j \in \Gamma_{i}^{+}} a_{i j} x_{1}^{j}+\sum_{j \in \Gamma_{i}^{+}} a_{i j} w_{j}-b_{2}^{i} .
\end{aligned}
$$

Therefore, the minimization problem (24) is changed to:

$$
\begin{cases}\min & Z=\frac{1}{4} \sum_{i=1}^{n}\left[\left(\Omega_{1}^{i}\right)^{2}+\left(\Omega_{2}^{i}\right)^{2}+\left(\Omega_{3}^{i}\right)^{2}+\left(\Omega_{4}^{i}\right)^{2}\right], \\ \text { s.t. } & x_{1}^{i} \leqslant \frac{1}{2} w_{i}, \\ & \alpha_{x}^{i} \geqslant \max \left\{0, \alpha_{x}^{* i}\right\} \\ & x_{1}^{i}: \text { free }, \quad i=1,2, \ldots, n .\end{cases}
$$

By the following theorem, we simplify the minimization problem (33).

Theorem 4.4. For any $1 \leqslant i \leqslant n, \Omega_{1}^{i}=-\Omega_{2}^{i}$ and $\Omega_{3}^{i}=-\Omega_{4}^{i}$. 
Proof. We have

$$
A W=C,
$$

then

$$
\sum_{j=1}^{n} a_{i j} w_{j}=\left(b_{1}^{i}+b_{2}^{i}\right)
$$

or

$$
\sum_{j \in \Gamma_{i}^{-}} a_{i j} w_{j}-b_{1}^{i}=b_{2}^{i}-\sum_{j \in \Gamma_{i}^{+}} a_{i j} w_{j}
$$

Now we have

$$
\begin{aligned}
\Omega_{1}^{i} & =\sum_{j \in \Gamma_{i}^{+}} a_{i j}\left(x_{1}^{j}-\alpha_{x}^{j}\right)-\sum_{j \in \Gamma_{i}^{-}} a_{i j}\left(x_{1}^{j}-\alpha_{x}^{j}\right)+\sum_{j \in \Gamma_{i}^{-}} a_{i j} w_{j}-\left(b_{1}^{i}-\alpha_{b}^{i}\right) \\
& =-\left[-\sum_{j \in \Gamma_{i}^{+}} a_{i j}\left(x_{1}^{j}-\alpha_{x}^{j}\right)+\sum_{j \in \Gamma_{i}^{-}} a_{i j}\left(x_{1}^{j}-\alpha_{x}^{j}\right)-\sum_{j \in \Gamma_{i}^{-}} a_{i j} w_{j}+b_{1}^{i}-\alpha_{b}^{i}\right] \\
& =-\left[-\sum_{j \in \Gamma_{i}^{+}} a_{i j}\left(x_{1}^{j}-\alpha_{x}^{j}\right)+\sum_{j \in \Gamma_{i}^{-}} a_{i j}\left(x_{1}^{j}-\alpha_{x}^{j}\right)+\sum_{j \in \Gamma_{i}^{+}} a_{i j} w_{j}-\left(b_{2}^{i}+\alpha_{b}^{i}\right)\right] \\
& =-\Omega_{2}^{i} .
\end{aligned}
$$

Similarly, since $A W=C$, then $\Omega_{3}^{i}=-\Omega_{4}^{i}$.

According to Theorem 4.3, we can simplify the minimization problem (33) as follows:

$$
\begin{cases}\min & Z=\frac{1}{2} \sum_{i=1}^{n}\left[\left(\Omega_{1}^{i}\right)^{2}+\left(\Omega_{3}^{i}\right)^{2}\right], \\ \text { s.t. } & x_{1}^{i} \leqslant \frac{1}{2} w_{i}, \\ & \alpha_{x}^{i} \geqslant \max \left\{0, \alpha_{x}^{* i}\right\}, \\ & x_{1}^{i}: \text { free } \quad i=1,2, \ldots, n .\end{cases}
$$

The minimization problem (25) has $2 n$ free variables, while the minimization problem (34) has $n$ free variables. Thus, solving (34) is more convenient than (25). Solving (34) and using the Eq. (20) we can obtain the symmetric $L$ $L$ fuzzy vector solution. If the optimal value of the the minimization problem, i.e. $Z$, was zero, then we get the exact solution of the S- $L$-FLS (7). Otherwise, If $Z$ was nonzero, then we get the approximate solution of the $S$-L-FLS (7). Note that, in the second case, the S- $L$-FLS (7) has not exact solution. In the following, we propose the first algorithm of our method to solve the S- $L$-FLS (7). Since the necessary condition for that an FLS has unique solution is that the matrices $A$ and $|A|$ are nonsingular [16], thus in this algorithm, we suppose 
that the matrices $A$ and $|A|$ are nonsingular.

\section{Algorithm 1}

Step 1: Construct and solve the crisp linear systems (19) and get the vectors $W$ and $\Lambda_{x}^{*}$.

Step 2: Construct the Eqs. (29) and (31) where $\alpha_{x}^{j}$ is replaced by $\alpha_{x}^{* j}$, and solve the minimization problem (34) and get $Z, x_{1}^{i}$ and $\alpha_{x}^{i}$ for $i=1,2, \ldots, n$.

Step 3: By Eq. (20) construct the symmetric $L-L$ fuzzy vector $\widetilde{X}=$ $\left(\widetilde{x_{1}}, \widetilde{x_{2}}, \ldots, \widetilde{x_{n}}\right)^{T}$.

Step 3: If $Z=0$ then $\widetilde{X}$ is an exact solution, otherwise $\widetilde{X}$ is an approximate solution.

\subsection{The second algorithm}

In this section, we suppose that the symmetric $L-L$ fuzzy vector $\widetilde{X}=\left(\widetilde{x_{1}}, \widetilde{x_{2}}, \ldots\right.$, $\left.\widetilde{x_{n}}\right)^{T}$ is the exact solution of S-L-FLS (7). Then, in the non-linear programming problem (34), we have

$$
Z=0,
$$

thus

$$
\Omega_{1}^{i}=0, \quad \Omega_{3}^{i}=0,
$$

and consequently

$$
|A| R=K, \quad|A| X_{1}=M,
$$

respectively, where

$$
\begin{array}{r}
(R)_{i}=x_{1}^{i}-\alpha_{x}^{i}, \quad(K)_{i}=\left(b_{1}^{i}-\alpha_{b}^{i}\right)-\sum_{j \in \Gamma_{i}^{-}} a_{i j} w_{j}, \\
\left(X_{1}\right)_{i}=x_{1}^{i}, \quad(M)_{i}=b_{1}^{i}-\sum_{j \in \Gamma_{i}^{-}} a_{i j} w_{j},
\end{array}
$$

for $i=1,2, \ldots, n$.

Finally, we set $\Lambda_{x}=X_{1}-R$ and $\Lambda_{b}=M-K$. Then, from (35)-(37), we have

$$
|A| \Lambda_{x}=\Lambda_{b}, \quad|A| X_{1}=M,
$$

where

$$
\left(\Lambda_{x}\right)_{i}=\alpha_{x}^{i}, \quad\left(\Lambda_{b}\right)_{i}=\alpha_{b}^{i},
$$


and

$$
\left(X_{1}\right)_{i}=x_{1}^{i}, \quad(M)_{i}=b_{1}^{i}-\sum_{j \in \Gamma_{i}^{-}} a_{i j} w_{j}
$$

for $i=1,2, \ldots, n$.

Based on the systems (19) and (38) and the minimization problem (34) and also Eq. (20), we propose the our second algorithm. Similarly to Algorithm 1 , we suppose that the matrices $A$ and $|A|$ are nonsingular.

\section{Algorithm 2}

Step 1: Construct and solve the crisp linear systems (19) and (38) and get the vectors $W, \Lambda_{x}, X_{1}$.

Step 2: By Eq. (20) get the vector $X_{2}=\left(x_{2}^{1}, x_{2}^{2}, \ldots, x_{2}^{n}\right)^{T}$ and then construct the vector $\widetilde{X}=\left(\widetilde{x_{1}}, \widetilde{x_{2}}, \ldots, \widetilde{x_{n}}\right)^{T}$.

Step 3: If $\widetilde{X}$ is the symmetric L-L fuzzy number, i.e. $\alpha_{x}^{i} \geqslant 0$ and $x_{1}^{i} \leqslant x_{2}^{i}$, then $\widetilde{X}$ is an exact solution, otherwise the problem has not exact solution.

Step 4: To obtain the symmetric approximate solution, construct the Eqs. (29) and (31) and solve the minimization problem (34) and get $x_{1}^{i}$ and $\alpha_{x}^{i}$ for $i=1,2, \ldots, n$.

Step 5: By Eq. (20) get the vector $X_{2}=\left(x_{2}^{1}, x_{2}^{2}, \ldots, x_{2}^{n}\right)^{T}$ and then construct the symmetric $L-L$ fuzzy number vector $\widetilde{X}=\left(\widetilde{x_{1}}, \widetilde{x_{2}}, \ldots, \widetilde{x_{n}}\right)^{T}$ as a fuzzy symmetric approximate solution.

It should be noted that based on the Algorithm 2, if the S- $L$-FLS (7) has an exact solution, then we don't need to solve the minimization problem (34) for obtaining the solution.

\section{$5 \quad$ Numerical examples}

In this section, we fix the shape function $L(\cdot)$ as:

$$
L(x)=R(x)=\max \{0,1-x\}, \quad \forall x \in \mathbb{R}^{+} .
$$

Therefore, in the following examples, the considered fuzzy numbers are either triangular fuzzy numbers or trapezoidal fuzzy numbers. 
Example 5.1. Consider the $3 \times 3 S$ - $L-F L S$

$$
\left\{\begin{array}{l}
-\widetilde{x_{1}}-\widetilde{x_{2}}+\widetilde{x_{3}}=(2,7 ; 6,6), \\
2 \widetilde{x_{1}}+\widetilde{x_{2}}-2 \widetilde{x_{3}}=(-7,1 ; 11,11), \\
-3 \widetilde{x_{1}}+2 \widetilde{x_{2}}-\widetilde{x_{3}}=(-12,-3 ; 11,11) .
\end{array}\right.
$$

According to Algorithm 2, by solving systems $A W=C,|A| \Lambda_{x}^{*}=\Lambda_{b}$ and $|A| X_{1}=M$ we obtain

$$
W=(3,0,6)^{T}, \quad \Lambda_{x}^{*}=(2,1,3)^{T}, \quad X_{1}=(1,-1,2)^{T} .
$$

Also, from Eq. (20) we have

$$
X_{2}=\left(x_{2}^{1}, x_{2}^{2}, x_{2}^{3}\right)^{T}=(2,1,4)^{T} .
$$

Obviously $\alpha_{x}^{* i} \geqslant 0$ and $x_{1}^{i} \leqslant x_{2}^{i}$, for $i=1,2,3$. Therefore, the symmetric $L-L$ fuzzy vector

$$
\widetilde{X}=\left[\begin{array}{c}
\left(x_{1}^{1}, x_{2}^{1} ; \alpha_{x}^{1}, \alpha_{x}^{1}\right) \\
\left(x_{1}^{2}, x_{2}^{2} ; \alpha_{x}^{2}, \alpha_{x}^{2}\right) \\
\left(x_{1}^{3}, x_{2}^{3} ; \alpha_{x}^{3}, \alpha_{x}^{3}\right)
\end{array}\right]=\left[\begin{array}{c}
(1,2 ; 2,2) \\
(-1,1 ; 1,1) \\
(2,4 ; 3,3)
\end{array}\right]
$$

is obtained as the fuzzy exact solution for S- $L$ - FLS (39).

Example 5.2. Consider the $3 \times 3 S$ - $L$ - FLS

$$
\left\{\begin{array}{l}
\widetilde{x_{1}}-2 \widetilde{x_{2}}+\widetilde{x_{3}}=(-4,1 ; 5,5), \\
-\widetilde{x_{1}}-\widetilde{x_{2}}+\widetilde{x_{3}}=(-5,-2 ; 4,4), \\
-\widetilde{x_{1}}+\widetilde{x_{2}}+\widetilde{x_{3}}=(-2,-1 ; 6,6) .
\end{array}\right.
$$

According to Algorithm 2, by solving systems $A W=C,|A| \Lambda_{x}^{*}=\Lambda_{b}$ and $|A| X_{1}=M$ we obtain

$$
W=(4,4,1)^{T}, \quad \Lambda_{x}^{*}=(2,1,1)^{T}, \quad X_{1}=(3,1,-1)^{T} .
$$

Also, from Eq. (20) we have

$$
X_{2}=\left(x_{2}^{1}, x_{2}^{2}, x_{2}^{3}\right)^{T}=(1,3,2)^{T} .
$$


Obviously, since $x_{1}^{1} \not \leq x_{2}^{1}$ then $\widetilde{x_{1}}$ is not a fuzzy number. Consequently, the vector $\widetilde{X}$ is not a fuzzy vector and therefore the problem has not exact solution. To find the symmetric approximate solution, we must construct $\Omega_{1}^{i}$ and $\Omega_{3}^{i}$, for $i=1,2,3$, and solve the minimization problem (4.34). From Eqs. (29) and (31), we have

$$
\begin{gathered}
\Omega_{1}^{1}=2\left(x_{1}^{1}-\alpha_{x}^{1}\right)+2\left(x_{1}^{2}-\alpha_{x}^{2}\right)+\left(x_{1}^{3}-\alpha_{x}^{3}\right)+1, \\
\Omega_{1}^{2}=\left(x_{1}^{1}-\alpha_{x}^{1}\right)+\left(x_{1}^{2}-\alpha_{x}^{2}\right)+\left(x_{1}^{3}-\alpha_{x}^{3}\right)+1, \\
\Omega_{1}^{3}=2\left(x_{1}^{1}-\alpha_{x}^{1}\right)+\left(x_{1}^{2}-\alpha_{x}^{2}\right)+\left(x_{1}^{3}-\alpha_{x}^{3}\right), \\
\Omega_{3}^{1}=x_{1}^{1}+2 x_{1}^{2}+x_{1}^{3}-4, \\
\Omega_{3}^{2}=x_{1}^{1}+x_{1}^{2}+2 x_{1}^{3}-3, \\
\Omega_{3}^{3}=2 x_{1}^{1}+x_{1}^{2}+x_{1}^{3}-6 .
\end{gathered}
$$

Now, we must solve the problem

$$
\begin{cases}\min & Z=\frac{1}{2}\left[\left(\Omega_{1}^{1}\right)^{2}+\left(\Omega_{1}^{2}\right)^{2}+\left(\Omega_{1}^{3}\right)^{2}+\left(\Omega_{3}^{1}\right)^{2}+\left(\Omega_{3}^{2}\right)^{2}+\left(\Omega_{3}^{3}\right)^{2}\right], \\ \text { s.t. } & x_{1}^{1} \leqslant 2, \quad x_{1}^{2} \leqslant 2, \quad x_{1}^{3} \leqslant \frac{1}{2} \\ & \alpha_{x}^{1} \geqslant 2, \quad \alpha_{x}^{2} \geqslant 1, \quad \alpha_{x}^{3} \geqslant 1 .\end{cases}
$$

We solved the above minimization problem using GAMS software and obtain the optimal solution as follows:

$$
x_{1}^{1}=2, \quad x_{1}^{2}=0.8333, \quad x_{1}^{3}=0.5, \quad \alpha_{x}^{1}=2, \quad \alpha_{x}^{2}=1, \quad \alpha_{x}^{3}=1,
$$

with the optimal value

$$
Z=0.5833 \text {. }
$$

On the other hand, by Eq. (20), we have

$$
x_{2}^{1}=2, \quad x_{2}^{2}=3.1667, \quad x_{2}^{3}=0.5 .
$$

Obviously $\alpha_{x}^{i} \geqslant 0$ and $x_{1}^{i} \leqslant x_{2}^{i}$, for $i=1,2,3$. Therefore, the symmetric $L-L$ fuzzy vector

$$
\widetilde{X}=\left[\begin{array}{l}
(2,2 ; 2,2) \\
(0.8333,3.1667 ; 1,1) \\
(0.5,0.5 ; 1,1)
\end{array}\right]
$$

is a symmetric fuzzy approximate solution for S- $L$ - FLS (40) where

$$
d(A \widetilde{X}, \widetilde{B})=\sqrt{Z}=0.7637 .
$$


Moreover, we put obtained solution of S- $L$-FLS into the original S- $L$-FLS (40) to investigate the difference between value of row $i\left((A \widetilde{X})_{i}\right)$ and $i$ th element of right hand side $\widetilde{B}\left(\widetilde{b_{i}}\right)$. For more detail see Fig $2-4$.

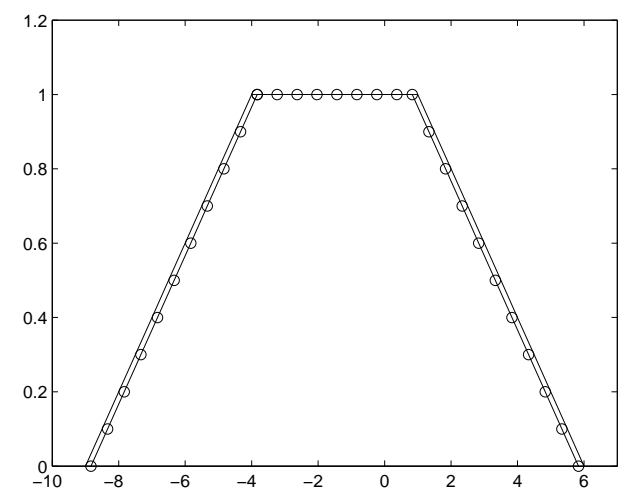

Fig. 2. Compare $\widetilde{b_{1}}(-)$ and value of first row, i.e. $(A \widetilde{X})_{1},(-\circ)$.

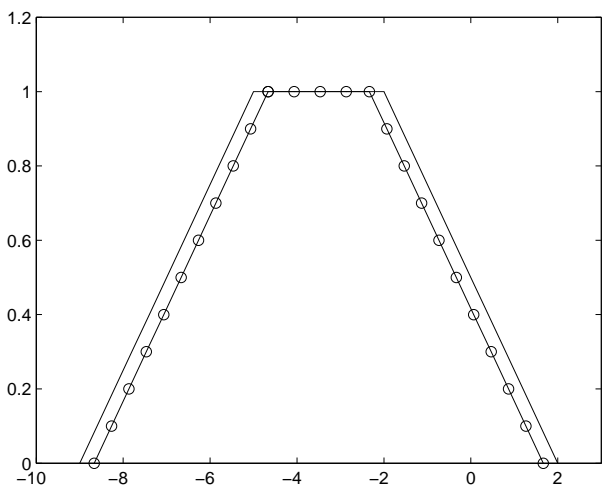

Fig. 3. Compare $\widetilde{b_{2}}(-)$ and value of second row , i.e. $(A \widetilde{X})_{2},(-\circ)$.

\section{Conclusion}

A new method for solving $S$ - $L$-FLS was introduced based on a metric distance on $L$ - $R$ fuzzy numbers. It is important that functions $L(\cdot)$ and $R(\cdot)$ are fixed functions. Finally, using the proposed distance a non-linear programming 


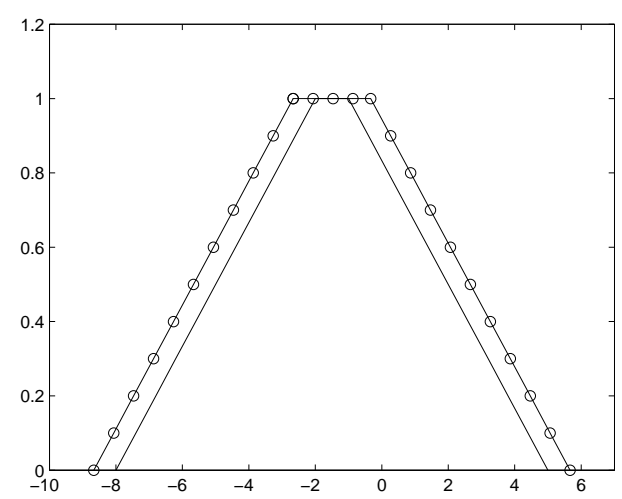

Fig. 4. Compare $\widetilde{b_{3}}(-)$ and value of third row, i.e. $(A \widetilde{X})_{3},(-\circ)$.

problem (NLP) was presented and solved. The constrains of NLP guarantees that the solution of $S$ - $L$-FLS is always an $L-L$ fuzzy number vector.

\section{References}

[1] S. Abbasbandy, R. Ezzati, A. Jafarian, LU decomposition method for solving fuzzy system of linear equations, Applied Mathematics and Computation, $\mathbf{1 7 2}(2006)$, 633-643.

[2] T. Allahviranloo, Numerical methods for fuzzy system of linear equations, Applied Mathematics and Computation, 155(2004), 493-502.

[3] T. Allahviranloo, M. Afshar Kermani, Solution of a fuzzy system of linear equation, Applied Mathematics and Computation, 175(2006), 519-531.

[4] T. Allahviranloo, M.A. Firozja, Ranking of fuzzy numbers by a new metric, Soft Computing, 14(2009), 773-782.

[5] T. Allahviranloo, M. Ghanbari, Solving Fuzzy Linear Systems by Homotopy Perturbation Method, International Journal of Computational Cognition, $\mathbf{8}(2)(2010), 24-30$.

[6] T. Allahviranloo, M. Ghanbari, A new approach to obtain algebraic solution of interval linear systems, Soft Computing, 16(2012), 121-133. 
[7] T. Allahviranloo, M. Ghanbari, On the algebraic solution of fuzzy linear systems based on interval theory, Applied Mathematical Modelling, (2012), DOI.org/10.1016/j.apm.2012.01.002.

[8] T. Allahviranloo, M. Ghanbari, A.A. Hosseinzadeh, E. Haghi, R. Nuraei, A note on "Fuzzy linear systems",Fuzzy Sets and Systems, 177 (1)(2011), 87-92.

[9] T. Allahviranloo, S. Khezerloo, M.A.Firozja, Fuzzy Regression on Fuzzy Data by New Metric Distance, Southeast Asian Bulletin of mathematics, 34(2010), 215-230.

[10] T. Allahviranloo, S. Salahshour, Fuzzy symmetric solution of fuzzy linear systems, Journal of Computational and Applied Mathematics, 235(16) (2011), 4545-4553.

[11] T. Allahviranloo, S. Salahshour, M. Khezerloo, Maximal- and minimal symmetric solutions of fully fuzzy linear systems, Journal of Computational and Applied Mathematics, 235(16) (2011), 4652-4662.

[12] M. Dehghan, B. Hashemi, Iterative solution of fuzzy linear systems, Applied Mathematics and Computation, 175(2006), 645-674.

[13] M. Dehghan, B. Hashemi, M. Ghatee, Computational methods for solving fully fuzzy linear systems, Applied Mathematics and Computation, 179(2006), 328343.

[14] P. Diamond, Fuzzy least squares, Information Sciences, 46(1988), 141-149.

[15] R. Ezzati, Solving fuzzy linear systems, Soft Computing, 15(2010), 193-197.

[16] M. Friedman, M. Ming and A. Kandel, Fuzzy linear systems, Fuzzy Sets and Systems, 96(1998), 209-261.

[17] R. Ghanbari, N. Mahdavi-Amiri, New solutions of L-R fuzzy linear systems using ranking functions and ABS algorithms, Applied Mathematical Modelling, 34(11) (2010), 3363-3375.

[18] R. Goetschel, W. Voxman, Elementary calculus, Fuzzy Sets and Systems, 18(1986), 31-43.

T. Allahviranloo,

Department of Mathematics, Science and Research Branch,

Islamic Azad University,

Tehran, Iran.

Email: Tofigh@Allahviranloo.com 


\section{E. Haghi,}

Department of Mathematics, Science and Research Branch,

Islamic Azad University,

Tehran, Iran.

Email: Haghi.Elnaz@yahoo.com

M. Ghanbari,

Department of Mathematics, Science and Research Branch, Islamic Azad University,

Tehran, Iran.

Email: Mojtaba.Ghanbari@gmail.com 
